\title{
Detection of anthropogenic objects based on the spatial characteristics of their contour in aerial image
}

\author{
Hayder Makki Hammed ${ }^{1}$, Osama Majeed Hilal Almiahi², Oksana Shauchuk ${ }^{3}$ \\ ${ }^{1}$ University of Baghdad, Iraq \\ ${ }^{2}$ Institute of Fine Arts, Directorate of Education in Ad Diwaniyah, Iraq \\ ${ }^{3}$ Department of Infocommunication Technologies, Belarusian State University of Informatics and Radioelectronics, \\ Belarus
}

\begin{abstract}
Article Info
Article history:

Received Jun 19, 2020

Revised Mar 21, 2021

Accepted Apr 6, 2021

Keywords:

Aerial images

anthropogenic objects

Geometric characteristics

Homogeneous regions

of the contour

Spatial characteristics

ABSTRACT

In this paper, an analysis is performed for the spatial characteristics of the contours of aerial images that are homogeneous in brightness to determine the parameters that make it possible to identify anthropogenic objects with a higher probability. A complex criterion is proposed for the regions, which homogenous in brightness and an algorithm for detecting anthropogenic objects, which takes into account its area, the ratio of the total length of long contour fragments to the total length of the contour, the concentration of corners and endpoints of the contour. Also, when deciding on anthropogenicity, the particular characteristics of the contour of a homogeneous segment are used, depending on the number of long fragments of the contour. Using the proposed criterion, an algorithm of searching anthropogenic objects based on the analysis of contour elements (its shape, size and concentration) is developed. The proposed algorithm allows reducing the probability of skipping the anthropogenic object in comparison with the spatial anomaly search algorithm.
\end{abstract}

This is an open access article under the CC BY-SA license.

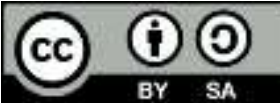

\section{Corresponding Author:}

Hayder Makki Hammed

University of Baghdad

Iraq

Email: haidermakki300@yahoo.com

\section{INTRODUCTION}

Currently, there is an intensive development of geographic information technologies [1], [2]. The range of tasks solved by geographic information systems (GIS) is expanding, and also increases the level of decision-making automation [3]-[8]. In connection with the development of unmanned aerial systems, GIS is becoming increasingly important, focused on aerial images formed using unmanned aerial vehicles (UAVs) [9]-[12]. Many of the tasks of modern GIS use the detection of anchor points in images of the earth's surface, moving objects, potential goals [13]-[17]. As a rule, such objects are anthropogenic (man-made), those have an unnatural origin. These are buildings [18]-[20], communications, altered soil, vehicles [21][23]. To implement the task of searching for anthropogenic objects in a GIS [24], methods from one or more of the following groups are most often used:

- Methods based on artificial neural networks [25], the disadvantage of these methods is high computational complexity, related the need for prior training.

- Methods based on the analysis of periodic textures [26] narrowly targeted methods, oriented detection of distributed objects (for example, city blocks), which does not allow them to be effectively used to search for concentrated anthropogenic objects (for example, roads, individual buildings). 
- Methods based on spectral parameters (vegetation index, moisture content index, shadow indicator) [27], requiring multispectral cameras on the side of the air complex.

- Methods based on spatial parameters (characteristics of the surroundings for the key points in the image) [28], using mainly the search for anomalies (deviations) in the distribution of brightness in aerial images, which in many cases leads to ambiguous decisions.

To eliminate the considered disadvantages, it is necessary to use a set of geometric characteristics (parameters) of an anthropogenic object in the spatial domain, which will allow them to be sampled on aerial images. Aim of the work: to develop an algorithm for detecting anthropogenic objects in the aerial image based on the analysis of the geometric characteristics of the contours of homogeneous regions.

\section{RESEARCH METHOD}

\subsection{Characteristics of the contours of the anthropogenic objects}

To analyze the characteristics of 4 types of anthropogenic objects, fragments of aerial images were used, received from UAVs on high $H=400$ meters at focal length $F=1920$ pixels (pixel size on the ground). Examples of test fragments are presented in Figure 1.

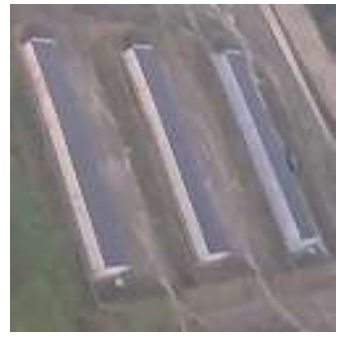

(a)

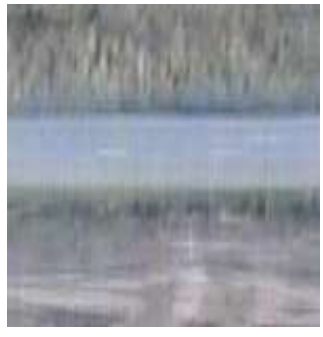

(b)

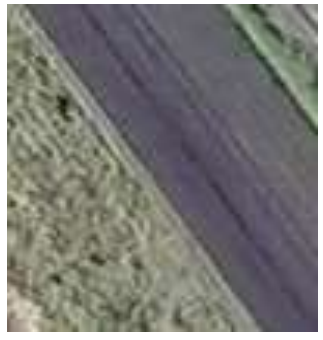

(c)

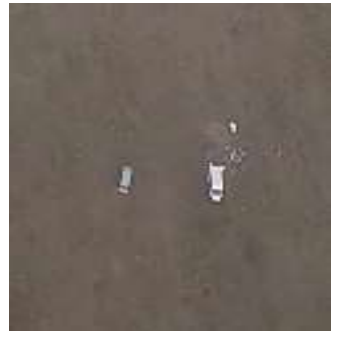

(d)

Figure 1. Test fragments of aerial images with various types of anthropogenic objects, (a) buildings,

(b) communications, (c) altered soil, (d) vehicles

For each test fragment of the aerial image, segmentation was performed using the algorithm regions growing [29]. At the same time, pixels were used as the key, containing in the Moore region more than 2 pixels, not exceeding the threshold difference $\mathrm{p}=2$ brightness components relative to the key. As a result of segmentation received overlapping segments to each test of aerial image. Segments considered if its dimensions s satisfy the following condition:

$$
\left\{\begin{array}{c}
s_{r}>2 \mathrm{~m}^{2}, \\
s=\frac{s_{r}}{G S P},
\end{array}\right.
$$

where $s_{r}$ - the real size of the object, $M^{2}$.

For each segment was performed High-pass filtering using Canny filter [30] (use characteristics: gradient search mask $5 \times 5$ pixels, upper and lower thresholds - 0 and 1 , accordingly, since a binary image was fed to the input) and thinning the obtained contours using the algorithm NCLT [31], [32]. Based on the fact, that in the contour can be distinguished such elements, as lines, angles and endpoints, in each of them the following parameters are determined that are essential for the detection of anthropogenic objects:

- Number of contour fragments;

- Length of each contour fragment;

- Number of the endpoints for each contour fragment;

- Number of angles for each contour fragment (100).

Examples for obtained segments and their corresponding contour with located end and corner points for each type of test aerial images are presented in Table 1. The analysis for the characteristics of the contours of anthropogenic objects as shown in Table 1 identified the following parameters:

- Smooth contour, consisting of a small number of fragments;

- Lack of accumulation of small fragments in the contour;

- Lack of a large number of angles and their concentration in the contour;

- Lack of a large number of endpoints and their concentration in the contour.

Detection of anthropogenic objects based on the spatial characteristics of their... (Hayder Makki Hammed) 
Table 1. Characteristics of the contour segment of aerial images with different types of objects

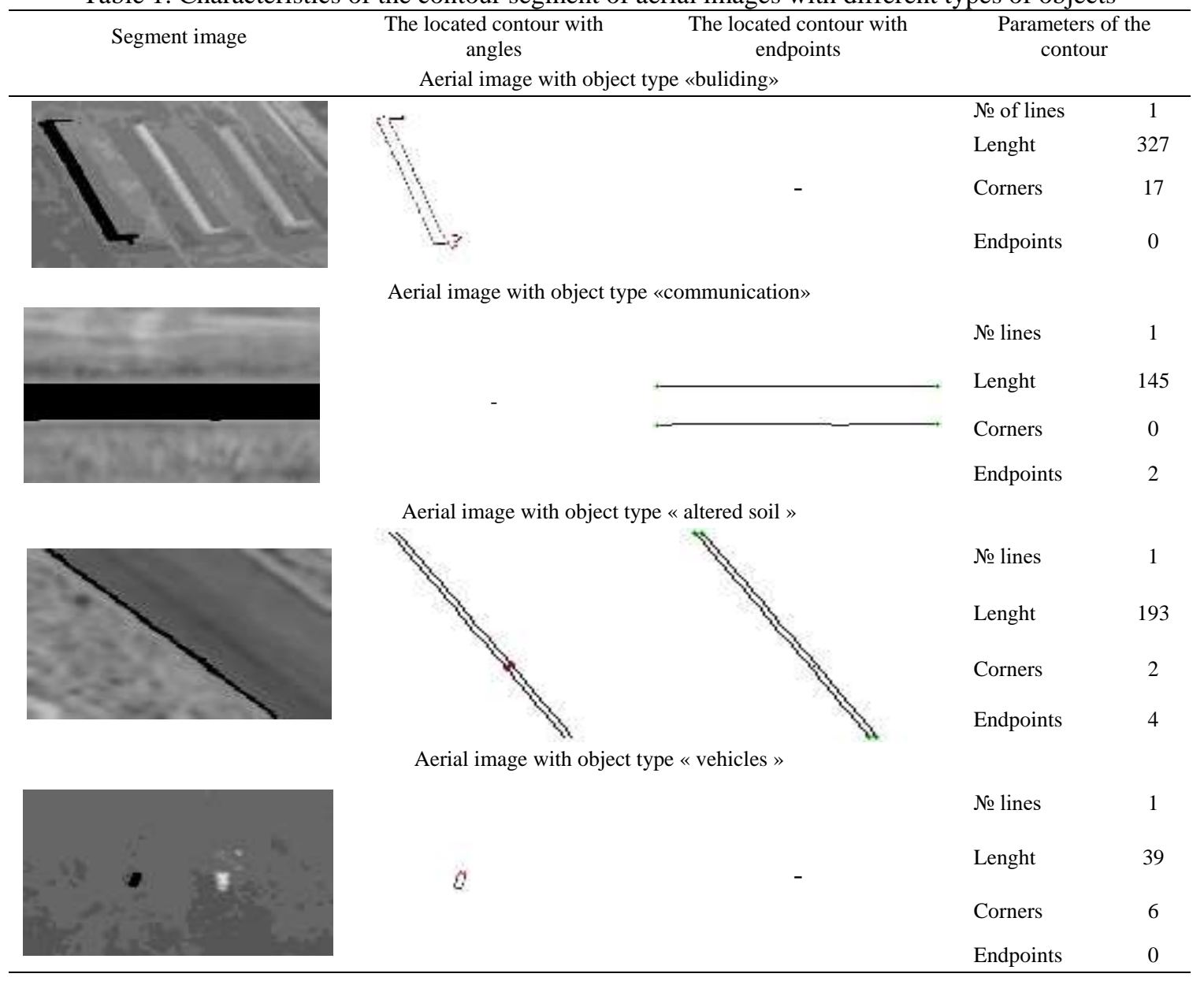

\subsection{A complex criterion for a homogeneous region in the brightness to anthropogenic objects}

Based on the presented characteristics of the contour segments of the aerial images with objects of different types Table 1, a complex criterion of a homogeneous region in brightness to anthropogenic objects has been formed based on shape analysis, size and concentration of its contour elements, which includes the following characteristics:

- The region s for a segment, which is homogeneous in brightness, should be more than 10 pixels function 1.

- The number of the selected contour fragments of the aerial image should fall in the interval: $K_{0} \in[1,50]$. Of these, the length of the contour fragments must satisfy the condition: $K_{L_{b}} \in[1,50]$. In this case, the contour fragment is understood as a fragment, the size which exceeds the value $t h r_{L} \in[9,11]$ pixels.

- The ratio of the total size to the length of the contour fragment $L_{b}$ to the total number of pixels in it $L_{0}$ must satisfy the condition:

$$
\frac{L_{b}}{L_{0}}>t h r,
$$

where $t h r \in[0,1]$ threshold.

- The ratio of the total number of endpoints to the length of the contour fragment $E_{b}$ to their number $K_{L_{b}}$ must satisfy the condition:

$$
O_{E} \leq 3 \text {. }
$$

- If a segment is characterized by only one long contour line, when $K_{L_{b}}=1$, and lack of endpoints $\left(O_{E}=0\right)$, it can be attributed to anthropogenic.

If the endpoints are present $\left(O_{E} \leq 3\right)$ - segment can be attributed to anthropogenic, if the ratio for the number of angles for a long contour fragment $C_{b_{1}}$ to its pixel length $L_{b_{1}}$ satisfies the condition: 


$$
\left\{\begin{array}{l}
O_{C}=\frac{C_{b_{1}}}{L_{b_{1}}} \\
O_{C} \leq 0.1
\end{array}\right.
$$

- Where $K_{L_{b}}=2$ segment can be attributed to anthropogenic, if both lines are double-ended straight lines, if the number of the pixels less than $50 \%$, therefor, must satisfy the condition:

$$
\left\{\begin{array}{c}
E_{b_{1}}=2, \\
E_{b_{2}}=2, \\
O_{L}=\frac{L_{b_{1}}}{L_{b_{2}}}, \\
O_{L} \hat{I}[0.6,1.7],
\end{array}\right.
$$

where $L_{b_{1}}, L_{b_{2}}$-number of the pixels for two long contour fragments; $E_{b_{1}}, E_{b_{2}}-$ number of the endpoints for two long contour fragments.

- If the characteristics of the contour segment dose not satisfy steps 4 and 5, then it can be attributed to anthropogenic under the following conditions:

$$
\overline{O_{C}} \leq 0.1
$$

where $\overline{O_{C}}$ - arithmetic mean for stack value $C_{b_{i}} / L_{b_{i}}$, calculated by the formula,

$$
\overline{O_{C}}=\frac{\sum_{i=1}^{K_{L_{b}}} \frac{C_{b_{i}}}{L_{b_{i}}}}{K_{L_{b}}}
$$

The algorithm to analysis the segment of aerial image based on the proposed complex criterion are uses as initial data, Figure 2:

- Formation of stack for coordinates of contour fragments $l=\{X(n), Y(n)\}_{\left(n=\overline{1, N_{S}}\right)}$ homogeneous region in brightness of the aerial image, where $n=\overline{1, N_{S}}$ - sequence number of a fragment; $N_{S}$ - the number of contour fragments which has the same brightness;

- Length for the contour fragments $K_{L_{b}} \leftarrow 0$, where $X(n)=\{x(i)\}_{i=\overline{0, K-1}}, \quad Y(n)=\{y(i)\}_{i=\overline{0, K-1}}$ coordinates for the contour fragments; $K$-number of pixels in the contour fragments.

Algorithm analysis contour segment of the aerial image consists of the following steps:

1. Counting the length for the contour fragment (how many pixels in the contour fragment) $K_{L_{b}}$.

If the length for the contour fragment satisfies the condition:

$L(n)>t h r_{L}$,

Then this contour refers to a long fragment, otherwise - refers to a short fragment.

if $K_{L_{b}}<1$, then go to step 12 .

2. Analyzing to the total size of the contour fragment $L_{b}$.

If $\frac{L_{b}}{L_{0}}<t h r$, where $t h r \in[0,1]-$ threshold value, then go to the step 12 .

3. Initializing the counter for the loop $j \leftarrow 1$.

4. Determining the total number of the endpoints $E_{b}(j)$.

5. Calculating form factor $f(j)$ for contour line. Use direct detection contour lines algorithm based on Euclidean form factor [32]. If for $j$ contour line condition:

$$
\left\{\begin{array}{l}
|1-f(j)| \leq T_{c}, \\
T_{c}=0,2,
\end{array}\right.
$$

then go to step 6, otherwise go to step 5 .

6. Searching the angles for the contour fragment. For searching the angles for the contour fragment $C_{b}(j)$ using the algorithm for detecting the angles of contour lines based on expandable masks [33].

7. Setting the counter value $j \leftarrow j+1$.

8. Analyzing the counter value.

when $j>K_{b}$ then go to step 4 , otherwise go to step 9 . 
9. Calculate the ratio of the total number of endpoints to long contour fragments to their number $K_{L_{b}}$ :

$O_{E}=\frac{\sum_{j=1}^{K_{L_{b}}} E_{b}(j)}{K_{L_{b}}}$.

if $O_{E} \leq 3$, then go to step 10 , otherwise go to step12.

10. Calculating the characteristics of contour elements for the segment homogenous in brightness depending on the number of its long fragments $K_{L_{b}}$ and their previously defined parameters:

$$
\left\{\begin{array}{l}
\left(K_{L_{b}}=1 \wedge O_{E}=0\right) \Rightarrow \text { go to step } 11, \\
\left(K_{L_{b}}=1 \wedge O_{E} \neq 0\right) \Rightarrow \text { go to step } 10.1, \\
\left(K_{L_{b}}=2 \wedge(|1-f(1)| \leq 0,2 \wedge|1-f(2)| \leq 0,2)\right) \Rightarrow \text { go to step 10.2, } \\
\text { otherwise } \Rightarrow \text { go to step 10.3. }
\end{array}\right.
$$

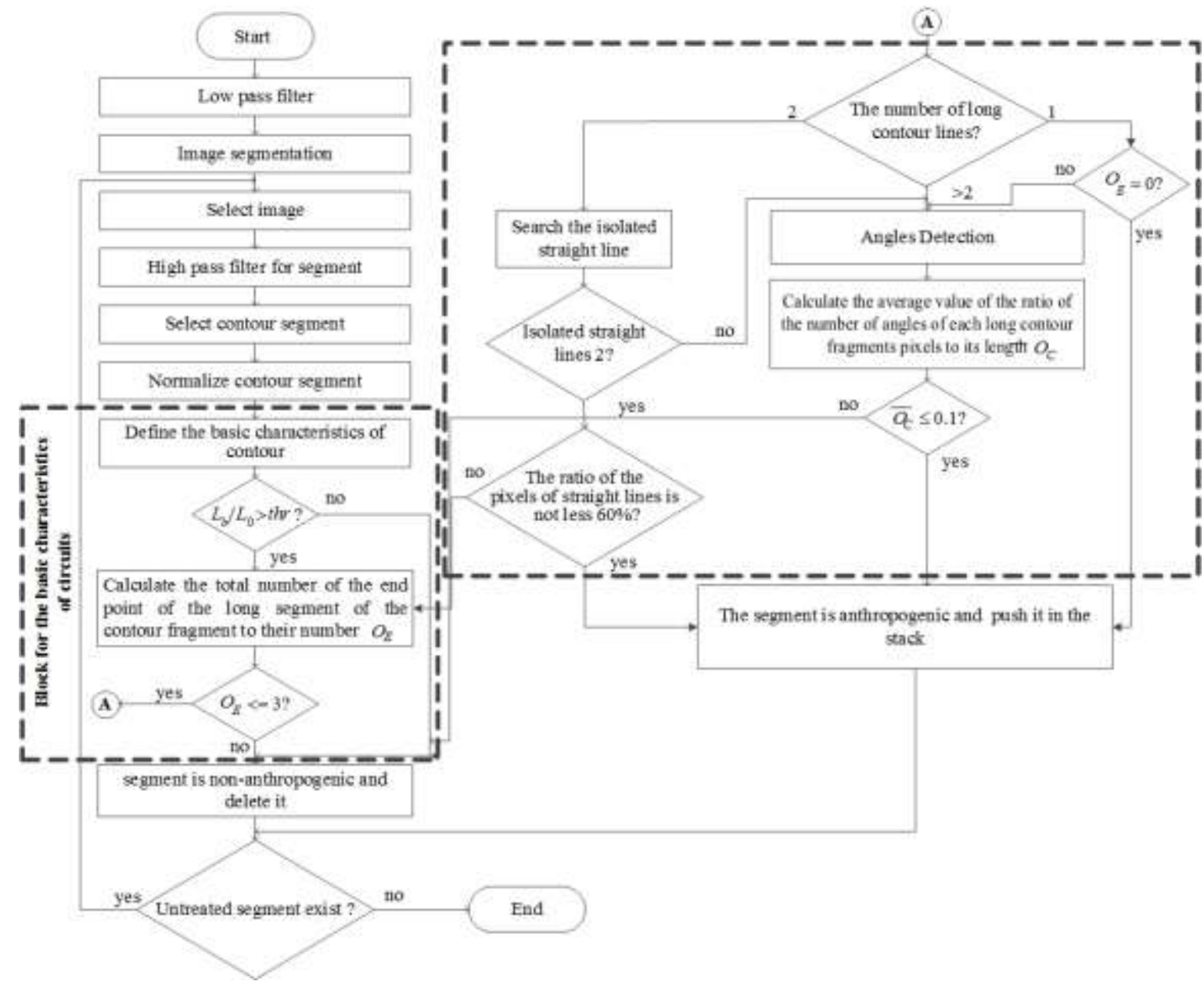

Figure 2. Algorithm of analysing the segment of aerial image based on the proposed complex criterion

10.1. Calculating the ratio of the number of angles for a long contour fragment $C_{b}(1)$ to its number of pixels in it $L_{b}(1)$ :

$$
O_{C}=\frac{C_{b}(1)}{L_{b}(1)}
$$


If $O_{C} \leq 0,1$, then go to step 11 , otherwise go to step 12 . each other:

10.2. Calculating the ratio of the number of the pixels for two long contour fragments relative to

$$
O_{L}=\frac{L_{b}(1)}{L_{b}(2)}
$$

where $L_{b}(1), L_{b}(2)$ - number of pixels in first and second contour fragment. If $0.6 \leq O_{L} \leq 1.7$, then go to step 11, otherwise go to step 12 .

10.3. Calculating $\overline{O_{C}}$. Use formula (2). If $\overline{O_{C}} \leq 0,1$, then go to step 11 , otherwise go to step 12 .

11. Deciding on the anthropogenic segment. The segment can be attributed to anthropogenic objects.

12. Ending for the algorithm. The segment can be attributed to non-anthropogenic objects.

As a result of the analysis of the aerial image segment on the basis of the proposed criterion, the selected homogenous region can be attributed to one of two types: anthropogenic or non-anthropogenic object.

\subsection{Algorithm detection of anthropogenic objects based on the spatial characteristics of their contour in aerial image}

Proposed algorithm (detection of anthropogenic objects based on the spatial characteristics of their contour in aerial image (DAOBSCC)). The developed algorithm DAOBSCC different from the known determination of affiliation for the homogenous regions in the brightness to anthropogenic objects, taking into account its area, the ratio of the total length for a long contour fragments to the total length of the contour, concentration of angles and endpoints of the contour.

The source of the data for the algorithm is a grayscale aerial image $I=i(y, x)_{(y=\overline{0, Y-1}, x=\overline{0, X-1})}$ Figure 3 (a).

Algorithm DAOBSCC consists of the following basic steps:

Step 1. Low pass filtering. Use Gaussian filter of size $S \times T$ with core

$$
w=\frac{1}{2 \pi \delta^{2}} \exp \left(-\frac{\left(s^{2}+t^{2}\right)}{2 \delta^{2}}\right),
$$

where $s \in\left[-\frac{(S-1)}{2}, \frac{(S-1)}{2}\right], t \in\left[-\frac{(T-1)}{2}, \frac{(T-1)}{2}\right]-$ coordinates convolution kernel abscissa and ordinate, $\delta-$ standard deviation of a Gaussian distribution.

At the output is formed a blurred grayscale image. $I_{g}=i_{g}(y, x)_{(y=\overline{0, Y-1}, x=\overline{0, X-1})}$ Figure $3(\mathrm{~b})$, where

$$
i_{g}(x, y)=\left\{\begin{array}{c}
\sum_{s=-\frac{S-1}{2}}^{\frac{(s-1)}{2}} \sum_{t=-\frac{T-1}{2}}^{\frac{(T-1)}{2}} w(s, t) i(x+s, y+t), \\
\text { where } y=\frac{T-1}{2}-1,(Y-1)-\frac{T-1}{2}, x=\frac{\overline{s-1}}{2}-1,(X-1)-\frac{s-1}{2}, \\
i(x, y), \text { otherwise. }
\end{array}\right.
$$
mask size:

Recommende the size for the mask $S=T=7$ pixels, deviation value - calculated according to

$$
\delta=0.3 \times((T-1) \times 0.5-1)+0.8
$$

Step 2. Segmentation. Image segmentation is performed using the region growing algorithm [34], [35]. In this case, pixels were used as key pixels, containing more than 3 pixels in the Moore region, with the difference in the brightness $p=2$.

As a result, a segmentation matrix is formed. $I_{s g}=\left\|i_{s g}(y, x, m)\right\|_{(y=\overline{0, Y-1}, x=\overline{0, X-1})}$, where $m=\left[1, M_{s g}\right] ; M_{s g}-$ the number of selected segments.

Segments smaller than 10 pixels are not considered Figure 3 (c).

Step 3. High pass filtering. For each $m$ segment matrix $I_{s g}$ use high-pass filtering by Ganny-edge detection algorithm. As a result, a binary matrix is formed $P=\|p(x, y, m)\|_{(y=\overline{0, Y-1}, x=\overline{0, X-1})}$ selected contours, where $p(x, y, m)=\{0,1\}$ - pixel value.

Step 4. Segmentation for contours of the aerial image (fragmentation). 
Use algorithm Brice and Fenema for contour segment in the matrix $P$, based on a selection of simple rules of growth and the binary value of the pixel, equal 1 .

As a result, is formed a segmentation matrix $I_{S}=\left\|i_{S}(y, x, m, n)\right\|_{(y=\overline{0, Y-1}, x=\overline{0, X-1})}$, where $n=\left[1, N_{s}(m)\right], N_{s}(m)-$ the number of selected contour segments.

Step 5. Thinning the contour fragment homogenous in the brightness for the segment of aerial images use algorithm thinning contour lines based on mask analysis of the local orientations of their fragments [31], [32]. As a result of the algorithm search and remove redundant pixels from matrices $I_{S}$.

The result of steps $3-5$ for the test image is shown in Figure $3(d)$.

Step 6. Selection and analysis for the basic characteristics of contour elements for a segment have the same brightness, use for this segment $l(m)=\{X(m, n), Y(m, n)\}_{\left(n=\overline{1, N_{S}(m)}\right)}$, where $X(m, n)=\{x(i)\}_{i=\overline{0, K-1}}, Y(m, n)=\{y(i)\}_{i=\overline{0, K-1}}-m, n$ coordinates contour fragment; $K$ - number of pixels in the counter fragment.

As a result of analysis characteristics contour elements of aerial image segments has formed a matrix of numbers of anthropogenic segments $M_{A}$.

As a result of the implementation algorithm based on matrix $M_{A}$ and $I_{s g}$ is formed stack for coordinate $I_{A}=\left\|i_{A}\left(y, x, m_{A}\right)\right\|_{(y=\overline{0, Y-1}, x=\overline{0, X-1})}$ segments of aerial images, describing found anthropogenic objects. The result of the proposed algorithm DAOBSCC for the test of aerial image shown in Figure 3 (e).

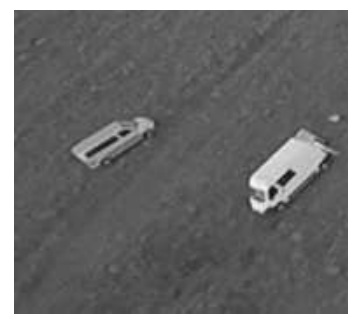

(a)

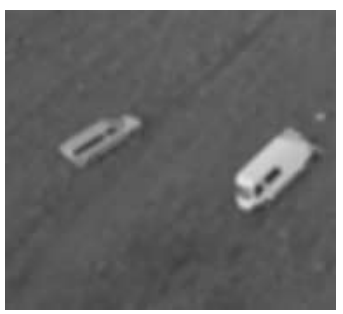

(b)

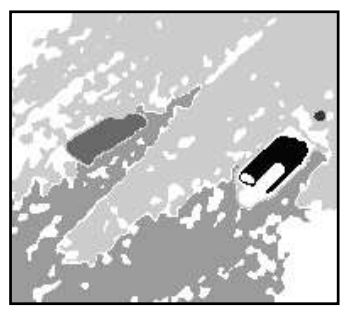

(c)

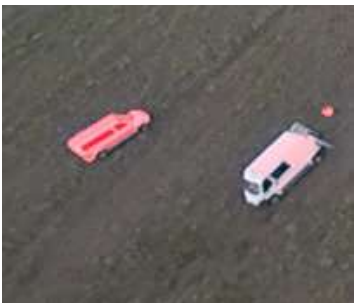

(e)

(d)

Figure 3. Step-by-step algorithm execution DAOBSCC, (a) original grayscale test aerial image,

(b) blurry test aerial image, (c) allocated aerial image segments, (d) the result of thinning the contours of allocated segments of the aerial image, and (e) allocated anthropogenic objects

\section{RESULTS AND DISCUSSION}

\subsection{Evaluation of the effectiveness of anthropogenic object detection algorithms}

The proposed algorithm is written in $\mathrm{C}++$. For a comparative assessment, an anthropogenic zone detection algorithm was used based on the search for spatial anomalies [5]. The experiment was tested in the computer with the following technical characteristics: processor Intel (R) Core (TM) i5-2320 CPU @ 3.0 GHz; RAM - 4 GB; Windows 7.

A study of the effectiveness for the proposed algorithm for the detection of anthropogenic objects was tested on aerial images, at the different times of the year with compact unmanned aerial vehicle (UAV) with the high $H=300 . .500$ meters, at focal length $F=1920$ pixels and the pixel size on the ground $G S D=0,16 . .0,26$. Five test sets were formed from these aerial images (50 images for each type), related to the following selected types of anthropogenic objects Figure 4:

- buildings Figure 4 (a);

- communications Figure $4(\mathrm{~b})$; 
- altered soil Figure 4 (c);

- vehicles Figure $4(\mathrm{~d})$;

- non-anthropogenic objects Figure 4 (e).

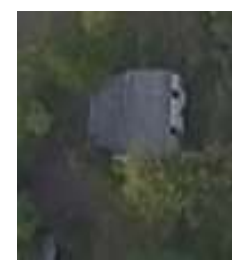

(a)

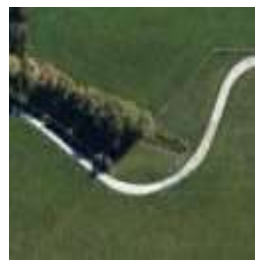

(b)

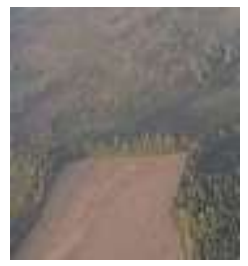

(c)

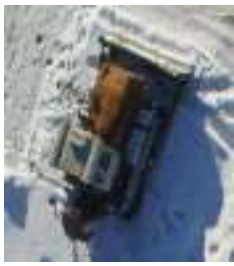

(d)

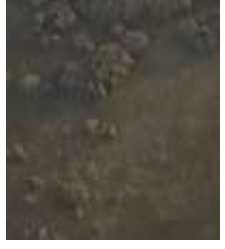

(e)

Figure 4. Step-by-step algorithm execution DAOBSCC, (a) buildings, (b) communications, (c) altered soil, (d) vehicles, (e) non-anthropogenic objects

For all fragments, anthropogenic objects were searched by algorithms DAZ [27] and DAOBSCC. Based on the obtained data, the effectiveness of these algorithms is estimated based on the calculation of the statistical probability of skipping $P_{1}$ anthropogenic object for each type. The statistical probability of skipping for each type of anthropogenic object was determined using the function:

$$
P_{1}=\frac{I_{e}}{I_{0}},
$$

where $I_{e}-$ number of aerial images with skipping anthropogenic objects;

$I_{0}-$ a total number of aerial images with a given type of anthropogenic object.

The results of a comparative analysis of algorithms for detecting anthropogenic objects on aerial images are presented in Table 2 . Table 2 shows, the proposed algorithm for detecting anthropogenic objects in comparison with DAZ allow reducing in the probability of skipping, 2.1 times for object type «building», 1.7 times - for object type «communication», 1.9 times - for object type « altered soil » and 2.3 times - for object type «vehicle».

Also, as criteria for the effectiveness of algorithms for the detection of anthropogenic objects in aerial images are used average $t_{a}$, minimum $t_{\min }$ and maximum $t_{\max }$ processing time. In Table 3 shows the processing time for the algorithms. From Table 3 shows, the proposed algorithm loses time compare with the algorithm DAZ by 2.1 times. At the time for the proposed algorithm a noticeable difference in the dispersion processing time-in some aerial images it faster than algorithm DAZ by 17.2 times.

Table 2. The statistical probability of skipping anthropogenic objects

\begin{tabular}{ccccc}
\hline \multirow{2}{*}{ Algorithms } & \multicolumn{4}{c}{$P_{1}$} \\
& Type 1 & Type 2 & Type 3 & Type 4 \\
\hline Proposed algorithm & 0.24 & 0.46 & 0.5 & 0.18 \\
DAZ & 0.5 & 0.76 & 0.94 & 0.42 \\
\hline
\end{tabular}

Table 3. Processing time for the algorithms, $\mathrm{s}$

\begin{tabular}{cccc}
\hline Algorithms & $t_{a}$ & $t_{\min }$ & $t_{\max }$ \\
\hline Proposed algorithm & 10.6 & 0.05 & 900 \\
DAZ & 5.1 & 0.86 & 73.3 \\
\hline
\end{tabular}

\section{CONCLUSION}

Developed algorithm detection of anthropogenic objects based on the spatial characteristics of their contour in the aerial image. The algorithm is differed from the known determination of affiliation for the homogenous regions in the brightness to anthropogenic objects, taking into account its area, the ratio of the total length for a long contour fragment to the total length of the contour, the concentration of angles and endpoints of the contour. The algorithm reduces the statistical probability of skipping anthropogenic objects by increasing the accuracy of taking into account their shape, size and concentration at the boundaries of regions that have the same brightness. Shown, the proposed algorithm allows to reduce the probability of skipping the anthropogenic object by 2.3 times in comparison with the spatial anomaly search algorithm with a decrease in processing time by 2.1 times. The disadvantages of the proposed algorithm include the dependence of the results on pre-processing (high-pass and low-pass filtering). 


\section{REFERENCES}

[1] R. Hartley and A. Zisserman, "Multiple view geometry in computer vision," Cambridge: Cambridge University Press, 2003, p. 655.

[2] E. Rosten and T. Drummond, "Machine learning for high-speed corner detection," Proceedings of the 9th Euproean Conference on Computer Vision, Austria, 7-13 May 2006. Austria, 2006, pp. 430-443.

[3] Q. Weng, "Remote Sensing and GIS Integration: Theory, Methods, and Applications," McGraw-Hill Education, 2009 , p. 416.

[4] F. Shahab, "GIS basic," New Delhi: New Age International, 2008, p. 354.

[5] O. Huisman, "Principles of geographic information systems (GIS): an introductory textbook," The Netherlands: ITC Educational Textbook Series, 2009, p. 540.

[6] G. Konecny, "Geoinformation: remote sensing, photogrammetry and geographic information systems," London: Taylor \& Francis, 2003, p. 248, doi: 10.1201/b12636.

[7] J. Leitloff, S. Hinz and U. Stilla, "Vehicle detection in very high resolution satellite images of city areas," IEEE Transactions on Geoscience and Remote Sensing, vol. 48, no. 7, pp. 2795-2806, 2010, doi: 10.1109/TGRS.2010.2043109.

[8] G. Cheng and J. Han, "A survey on object detection in optical remote sensing images," ISPRS Journal of Photogrammetry and Remote Sensing, vol. 117, pp. 12-28, 2016, doi: 10.1016/j.isprsjprs.2016.03.014.

[9] T. Blaschke, et al., "Geographic object-based image analysis-towards a new paradigm," Journal of Photogrammetry and Remote Sensing, vol. 87, no. 1, pp. 180-191, 2014, doi: 10.1016/j.isprsjprs.2013.09.014.

[10] D. Contreras, et al., "Monitoring recovery after earthquakes through the interation of remote sensing, GIS, and ground observations: the case of L'Aquila (Italy)," Cartography and Geographic Information Science, vol. 43, no. 2, pp. 115-133, 2015, doi: 10.1080/15230406.2015.1029520.

[11] A. Huertas and R. Nevatia, "Detecting buildings in aerial images," Computer Vision, Graphics, and Image Processing, vol. 41, pp. 131-152, 1988, doi: 10.1016/0734-189X(88)90016-3.

[12] H.G. Aksay and S. Aksoy, "Building detection using directional spatial constraints," Proceedings of the International Conference on Geoscience and Remote Sensing Symposium, Honolulu, HI, USA, 25-30 July 2010. USA, 2010, pp. 1932-1935, doi: 10.1109/IGARSS.2010.5652842.

[13] A. O. Ok, "Automated detection of buildings from single VHR multispectral images using shadow information and graph cuts," Journal of Photogrammetry and Remote Sensing, vol. 86, pp. 21-40, 2013, doi: 10.1016/j.isprsjprs.2013.09.004.

[14] S. Ahmadi, et al., "Automatic urban building boundary extraction from high resolution aerial images using an innovative model of active contours," International Journal of Applied Earth Observation and Geoinformation, vol. 12, no. 3, pp. 150-157, 2010, doi: 10.1016/j.jag.2010.02.001.

[15] S. Ahmadi, et al., "Automatic Urban Building Boundary Extraction From High Resolution Aerial Images Using An Innovative Model of Active Contours," International Journal of Applied Earth Observation and Geoinformation, no. 12, pp. 150-157, 2010, doi: 10.1016/j.jag.2010.02.001.

[16] S. Nebiker, N. Lack, and M. Deuber, "Building Change Detection From Historical Aerial Photographs Using Dense Image Matching And Object-Based Image Analysis," Remote sensing, no. 6, pp. 8310-8336, 2014, doi: $10.3390 /$ rs6098310.

[17] T. R. Martha, et al., "Characterising spectral, spatial and morphometric properties of landslides for semi-automatic detection using object-oriented methods," Geomorphology, vol. 116, pp. 24-36, 2010, doi: 10.1016/j.geomorph.2009.10.004.

[18] R. Chen, X. Li, and J. Li, "Object-Based Features For House Detection From Rgb High-Resolution Images," Remote Sensing, vol. 10, no. 451, pp. 1-24, 2018, doi: 10.3390/rs10030451.

[19] H. Alzakki, and V. Tsviatkou, "Texture image segmentation based on classification of contour elements and logical addition of classes," 2016 Al-Sadeq International Conference on Multidisciplinary in IT and Communication Science and Applications (AIC-MITCSA) (IEEE Conference Publications), vol. 24, pp. 1-6, 2016, doi: 10.1109/AIC-MITCSA.2016.7759922.

[20] V. venkateswar, and R. Chellappa, "Extraction of Straight Lines in Aerial Images," IEEE Trans. Pattern Analysis and Machine Intelligence, vol. 14, no. 11, pp. 1111-1114, 1992, doi: 10.1109/34.166627.

[21] C. Iovan, D. Boldo, and M. Cord, "Detection, Characterization, and Modeling Vegetation in Urban Areas From High-Resolution Aerial Imagery," Journal of Selected Topics in Applied Earth Observations and Remote Sensing, vol. 1, no. 3, pp. 206-213, 2008, doi: 10.1109/JSTARS.2008.2007514.

[22] K. Karantzalos, and D. Argialas, "A Region-based Level Set Segmentation for Automatic Detection of Man-made Objects from Aerial and Satellite Images," Photogrammetric Engineering \& Remote Sensing, vol. 75, no. 6, pp. 667-677, 2009, doi: 10.14358/PERS.75.6.667.

[23] A. Kovacs, and T. Sziranyi, "Orientation-Selective Building Detection in Aerial Images," Journal of Photogrammetry and Remote Sensing, vol. 108, pp. 94-112, 2015, doi: 10.1016/j.isprsjprs.2015.06.007.

[24] Y. T. Liow, and T. Pavlidis, "Use of Shadows for Extracting Buildings in Aerial Images," Computer Vision, Graphics, and Image Processing, vol. 49, pp. 242-277, 1990, doi: 10.1016/0734-189X(90)90139-M.

[25] V. Alexander, "Machine Learning Techniques in Task of Visual Recognition," Proceedings of the International Conference Graphicon, Novosibirsk Akademgorodok, Russia, 2006. Russia, 2006, pp. 87-92.

[26] S. Leninisha and K. Vani, "Water Flow Based Geometric Active Deformable Model for Road Network," Journal of Photogrammetry and Remote Sensing, vol. 102, no. 2, pp. 140-147, 2015, doi: 10.1016/j.isprsjprs.2015.01.013. 
[27] S. M. Borzov and A. O. Potaturkin, "Detection Of Anthropogenic Zones Based On Search Of Spatial Anomalies In Large-Scale Satellite Images," Novosibirsk State University, vol. 10, no. 5, pp. 86-90, 2013, doi: 10.3103/S8756699012050123.

[28] S. M. Borzov and A. O. Potaturkin, "Survey of Spatial Attributes' Effectiveness in Classification of Satellite Imagery of Various Resolutions," Novosibirsk State University, vol. 10, no. 3, pp. 58-65, 2012.

[29] F.Y. Shih and S. Cheng, "Automatic Seeded Region Growing for Color Image Segmentation," Image and Vision Computing, no. 23, pp. 877-886, 2005.

[30] J. Canny, "A Computational Approach to Edge Detection," IEEE Trans. Pattern Analysis and Machine Intelligence, vol. 8, no. 6, pp. 679-698, 1986, doi: 10.1109/TPAMI.1986.4767851.

[31] O. Shauchuk and V. Tsviatkou, "Method of Normalization of the Contour Line in Thickness Based On Binary Masks," Proceedings of Al-Sadeq International Conference on Multidisciplinary in IT and Communication Science and Applications, Baghdad, Iraq, 9-10 May 2016. Iraq, 2016, pp. 1-6, doi: 10.1109/AIC-MITCSA.2016.7759921.

[32] O. J. Al-Furaiji, A. G. Shauchuk, and V. Yu. Tsviatkou, "Localization And Normalization Of Isolated Direct Contour Lines Based On Masks And Form-Factor," Thi-Qar University Journal for Enginering Sciences, vol. 6, no. 3, pp. 1-18, 2015.

[33] O. G. Shevchuk and V. Y.Tsviatkou, "Detection Of Fracture A Contours On The Image Using Expanding Masks," Doklady BGUIR, no. 7, pp. 221-225, 2016.

[34] H. M. Alzakki, V. Yu. Tsviatkou, "Contour processing of texture Images," Proceedings of Second Engineering Scientific Conference, University of Diyala, Diyala, Iraq, 2015, pp. 40053-461.

[35] O. Almiahi and V. Kanapelka, "Progressive image segmentation based on the wave region growing," Proceedings of Al-Sadeq International Conference on Multidisciplinary in IT and Communication Science and Applications, Baghdad, Iraq, 2016, pp. 105-110, doi: 10.1109/AIC-MITCSA.2016.7759919.

\section{BIOGRAPHIES OF AUTHORS}

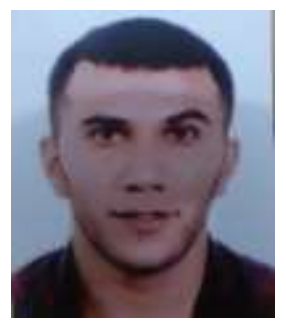

Hayder Makki Hammed Alzaki, University of Baghdad, Iraq. Ph. D. Degree from Belarusian State University of Informatics and Radioelctronics (BSUIR), Minsk, Belarus. Specialty: Telecommunication Systems, Networks and Devices. Email: haidermakki300@yahoo.com

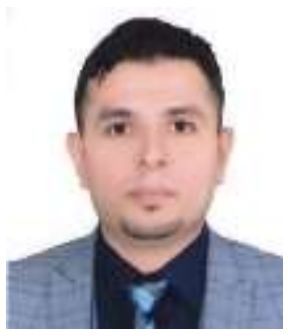

Osama Majeed Hilal Almiahi, Lecturer of the Institute of Fine Arts, Directorate of Education in Ad Diwaniyah, Ad-Diwaniyah, Iraq. Ph. D. Degree from Belarusian State University of Informatics and Radioelctronics (BSUIR), Minsk, Belarus. Specialty: Computer scienceInformation security. Email: almiahi86@yahoo.com

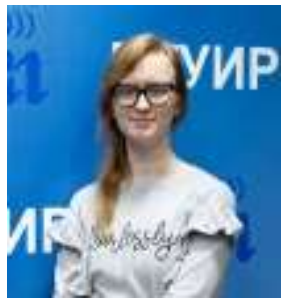

Oksana Shauchuk, Department of infocommunication technologies of Belerusian State University of Informatics and Radioelectronics (BSUIR). Ph. D. Degree from Belarusian State University of Informatics and Radioelctronics (BSUIR), Minsk, Belarus. Specialty: Telecommunication Systems, Networks and Devices. Email: vtsvet@bsuir.by 